\title{
Estimation of long-term groundwater storage variation in the Lower Transboundary Indus Basin using GRACE and WGHM model data
}

\author{
shoaib ali ${ }^{1}$, Qiumei Wang ${ }^{1}$, Dong Liu ${ }^{1}$, Qiang $\mathrm{Fu}^{1}$, Md. Mafuzur Rahaman ${ }^{2}$, Muhammad \\ Abrar Faiz ${ }^{1}$, and Muhammad Jehanzeb Masud Cheema ${ }^{3}$ \\ ${ }^{1}$ Northeast Agricultural University \\ ${ }^{2} \mathrm{AECOM}$ \\ ${ }^{3}$ Pir Mehr Ali Shah Arid Agriculture University
}

December 22, 2020

\begin{abstract}
In the Lower Transboundary Indus Basin (LTIB), excessive groundwater is being consumed in combination with surface water to meet the increasing demand of irrigation, resulting in groundwater depletion that needs to be quantified. This study used GRACE (Gravity Recovery And Climate Experiment) satellite terrestrial water storage anomalies (TWSA) and Global Land Data Assimilation System (GLDAS) model data to produce monthly groundwater storage anomalies (GWSA) and to evaluate the depletion of groundwater storage in the LTIB. It is observed that the variation in GWSA exhibits a downward trend from 2003 to 2016. Additionally, TWSA and precipitation data depict seasonal characteristics with peaks in the summer and dips in the winter, which reflect variation in GWSA, respectively. GRACE TWSA measurements also detected massive floods that occurred in 2010 and 2015, and they significantly recharged groundwater in the LTIB. This study also utilized Empirical Orthogonal Function (EOF) analysis to assess the variance variability. The results revealed that more than $80 \%$ of total variance variability was explained by the first 2 EOF modes. The generalized three-cornered hat method (GTCH) was used to estimate the uncertainty of different GRACE TWSA measurements. The results show that groundwater storage is being depleted at a rate of $4.16 \mathrm{~mm}$ per year $(2.97 \mathrm{~km} 3$ per year). Long-term monthly mean GRACE derived GWSA showed remarkable agreements with PCRaster Global Balance (PCR-GLOBWB) model 75\% and WGHM (WaterGap Global Hydrological model) $81 \%$. This study can be helpful to calculate the socio and agro-economic impact of the excessive withdrawal of groundwater.
\end{abstract}

\section{Hosted file}

Shoaib_Ali__manuscript.pdf available at https://authorea.com/users/384367/articles/500003estimation-of-long-term-groundwater-storage-variation-in-the-lower-transboundary-indusbasin-using-grace-and-wghm-model-data 

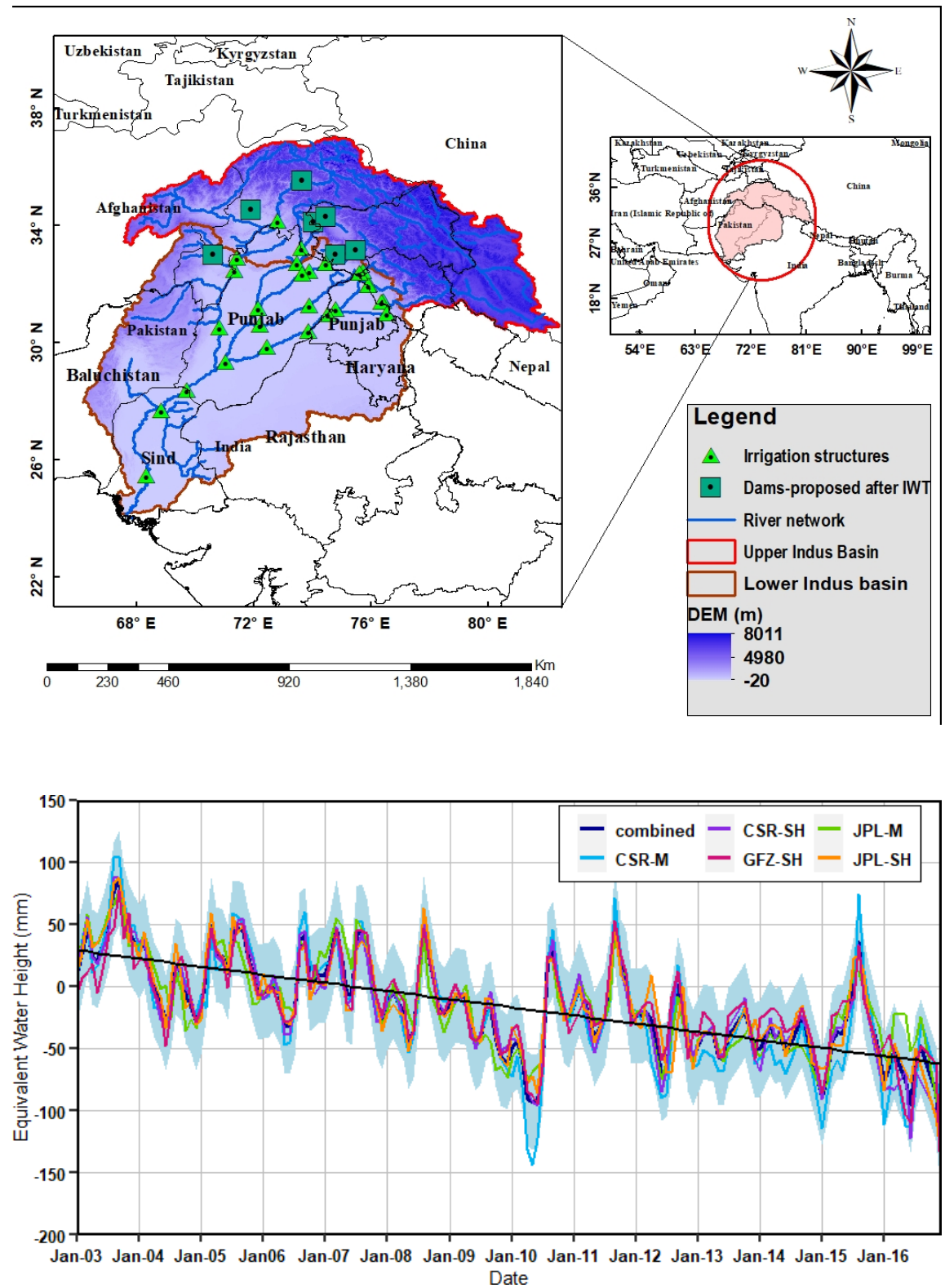

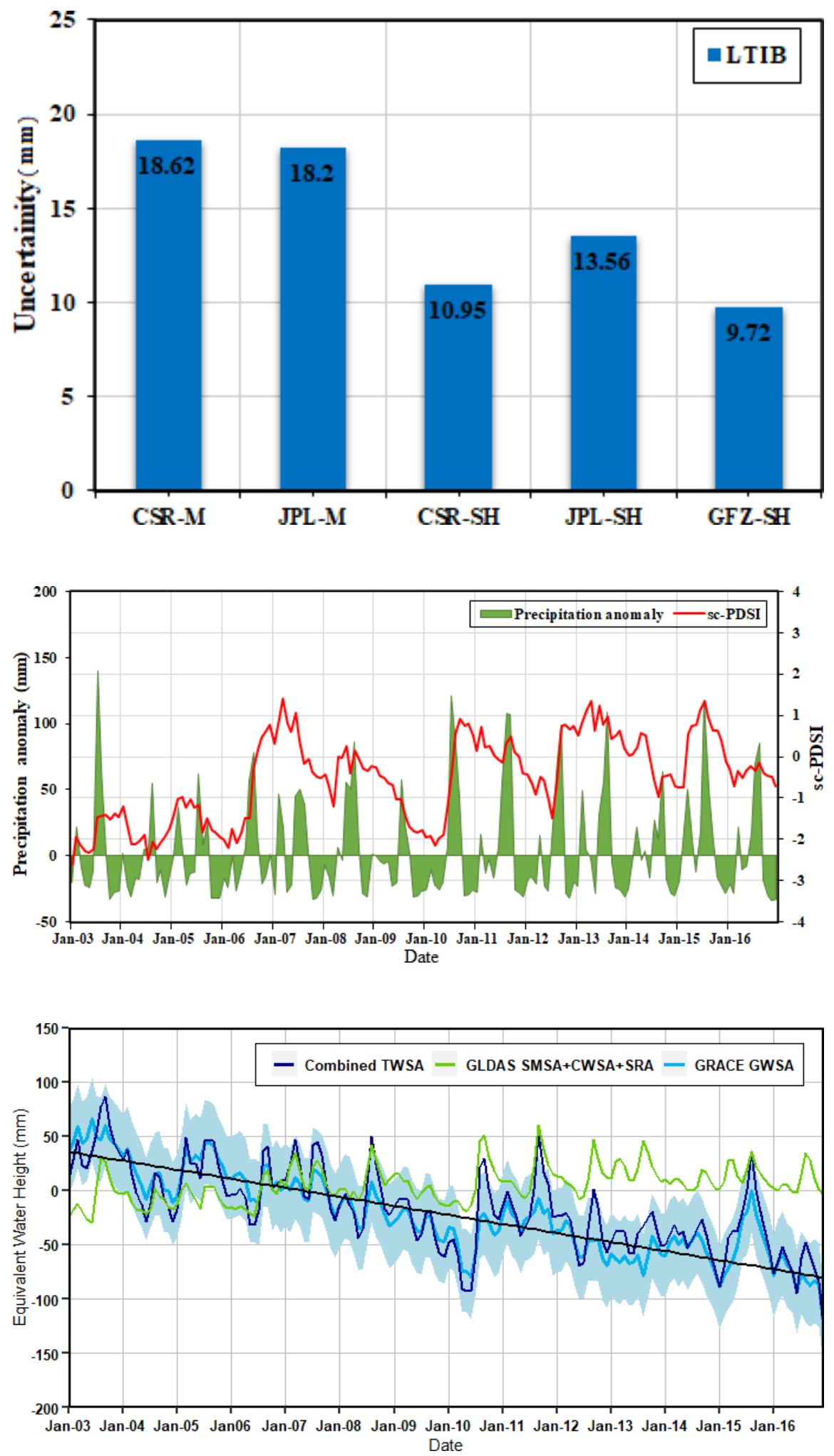

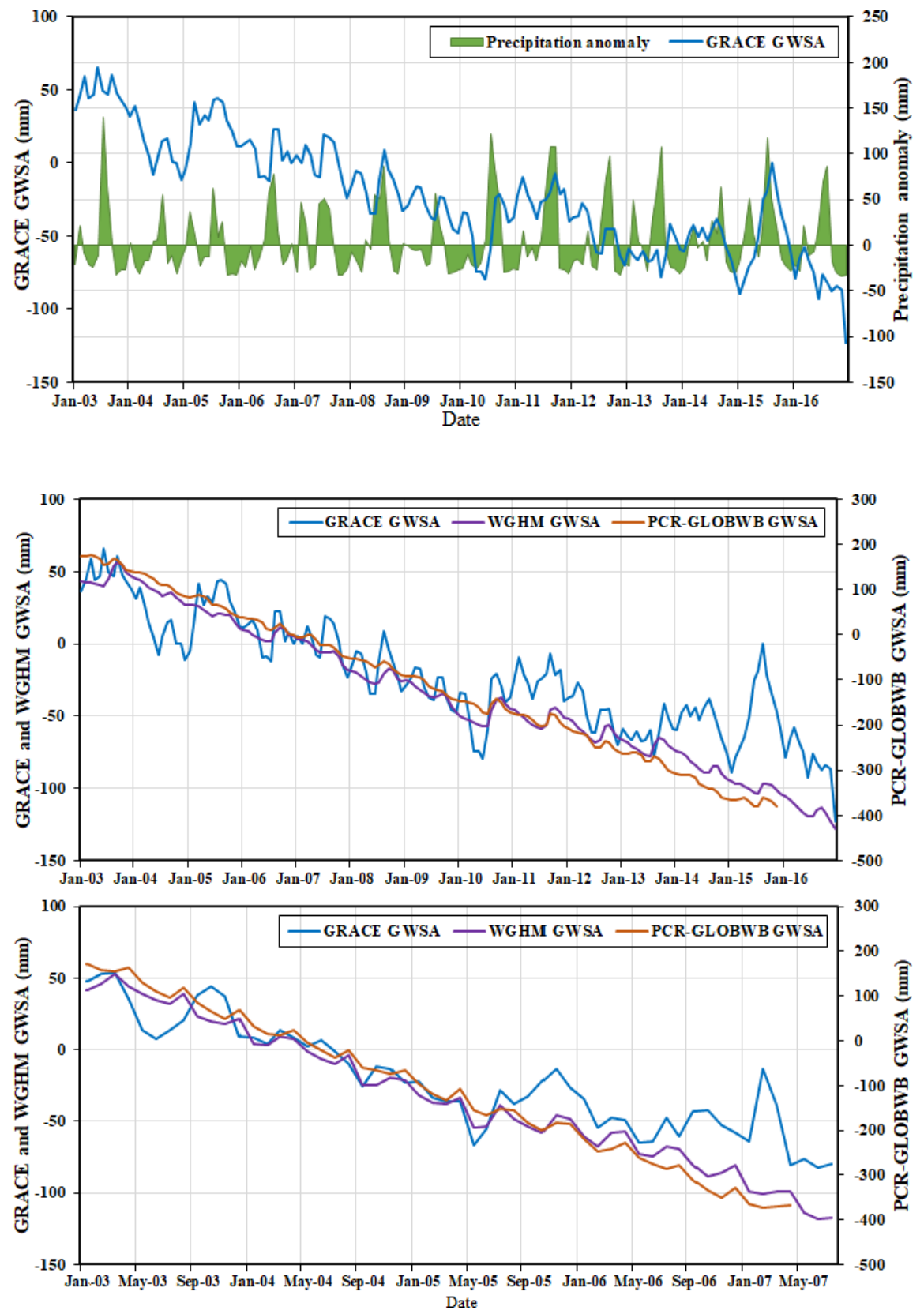





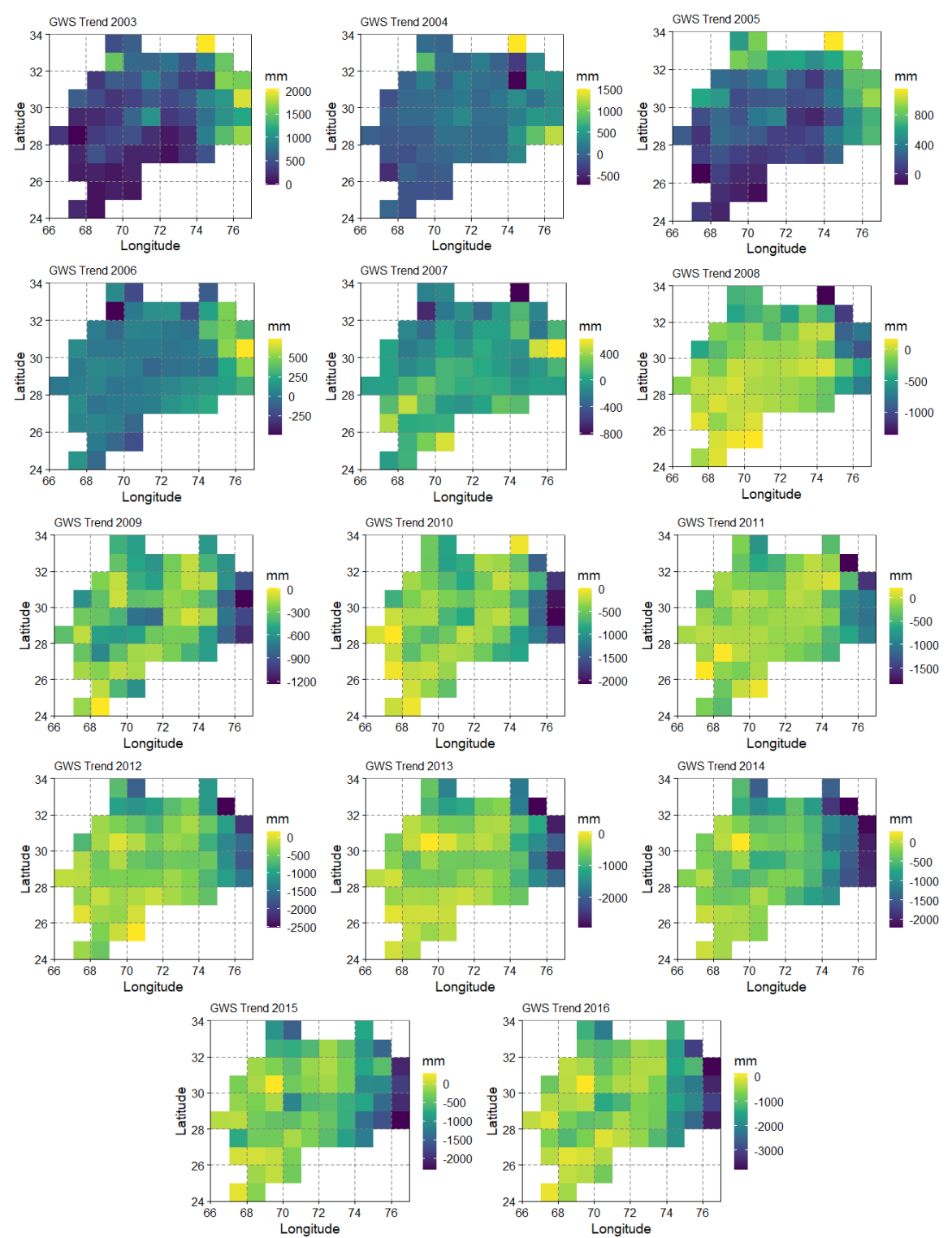

